\title{
Ankylosing Spondylitis Patients Commencing Biologic Therapy Have High Baseline Levels of Comorbidity: A Report from the Australian Rheumatology Association Database
}

\author{
John Oldroyd, ${ }^{1,2}$ Lionel Schachna, ${ }^{3}$ Rachelle Buchbinder, ${ }^{1,2}$ Margaret Staples, ${ }^{1,2}$ \\ Bridie Murphy, ${ }^{1,2}$ Molly Bond, ${ }^{4}$ Andrew Briggs, ${ }^{1,2,5}$ Marissa Lassere, ${ }^{6}$ and Lyn March ${ }^{7,8}$ \\ ${ }^{1}$ Monash Department of Clinical Epidemiology, Cabrini Institute, 183 Wattletree Road, Malvern, VIC 3144, Australia \\ ${ }^{2}$ Department of Epidemiology and Preventive Medicine, School of Public Health and Preventive Medicine, \\ Monash University, VIC 3004, Australia \\ ${ }^{3}$ Austin Spondylitis Clinic, Austin Health, Studley Road, Heidelberg, VIC 3084, Australia \\ ${ }^{4}$ Centre of Clinical Research Excellence in Therapeutics, Department of Epidemiology and Preventive Medicine, \\ School of Public Health and Preventive Medicine, Monash University, VIC 3800, Australia \\ ${ }^{5}$ School of Physiotherapy, Curtin University of Technology, WA 6000, Australia \\ ${ }^{6}$ Department of Rheumatology, St George Hospital, NSW 2217, Australia \\ ${ }^{7}$ Department of Rheumatology, Royal North Shore Hospital, NSW 2065, Australia \\ ${ }^{8}$ Institute of Bone and Joint Research, University of Sydney, NSW 2065, Australia
}

Correspondence should be addressed to Rachelle Buchbinder, rachelle.buchbinder@med.monash.edu.au

Received 13 April 2009; Accepted 28 May 2009

Recommended by Malcolm D. Smith

\begin{abstract}
Aims. To compare the baseline characteristics of a population-based cohort of patients with ankylosing spondylitis (AS) commencing biological therapy to the reported characteristics of bDMARD randomised controlled trials (RCTs) participants. Methods. Descriptive analysis of AS participants in the Australian Rheumatology Association Database (ARAD) who were commencing bDMARD therapy. Results. Up to December 2008, 389 patients with AS were enrolled in ARAD. 354 (91.0\%) had taken bDMARDs at some time, and 198 (55.9\%) completed their entry questionnaire prior to or within 6 months of commencing bDMARDs. $131(66.1 \%)$ had at least one comorbid condition, and $24(6.8 \%)$ had a previous malignancy (15 nonmelanoma skin, 4 melanoma, 2 prostate, 1 breast, cervix, and bowel). Compared with RCT participants, ARAD participants were older, had longer disease duration and higher baseline disease activity. Conclusions. AS patients commencing bDMARDs in routine care are significantly different to RCT participants and have significant baseline comorbidities.
\end{abstract}

Copyright (C) 2009 John Oldroyd et al. This is an open access article distributed under the Creative Commons Attribution License, which permits unrestricted use, distribution, and reproduction in any medium, provided the original work is properly cited.

\section{Introduction}

Ankylosing spondylitis is a chronic inflammatory rheumatic disorder predominantly affecting the spine with a reported prevalence in Caucasian populations varying between $0.05 \%$ to $0.23 \%$ [1]. AS has a substantial impact upon physical and emotional functioning and is associated with a progressive decline in quality of life $[2,3]$. In those with severe disease, mortality is increased [4]. Extraspinal features may include peripheral arthritis, uveitis, enteritis, and psoriasis. Traditional disease modifying antirheumatic drugs (DMARDs) have been ineffective for the spinal component of the disease with the mainstay of treatment consisting of exercise therapy [5] and nonsteroidal anti-inflammatory drugs $[6,7]$.

Recently, biological disease modifying antirheumatic drugs (bDMARDs) including the tumor necrosis factor (TNF alpha) inhibitors etanercept, infliximab, and adalimumab have been shown to reduce disease activity and results in improvements in pain, function, and quality of life in AS [8-12]. Randomized controlled trials (RCTs) have established large treatment benefits for spinal pain, mobility, and function as well as improvements in extraspinal features $[9,10,13]$. Whether these effects will be fully realized and maintained in routine care remains to be determined as 
the trials have been of relatively short duration (e.g., 2454 weeks) and have excluded important patient groups (i.e., patients under the age of 18 years and those with a history of cardiac, renal, hepatic, psychiatric, or neurologic disease or history of malignancy); and baseline comorbidities of trial participants have not been reported in detail [11, 14-16].

To address the limitations of clinical trials and determine the long-term safety and effectiveness of bDMARD therapy in AS in a "real life" context, population-based registries that allow careful longitudinal observation in routine clinical care have been established in several countries. These include the Australian Rheumatology Association Database (ARAD) [17], the British Society for Rheumatology Biologics Registry (BSRBR) [18], the Spanish Registry for adverse events of biological therapies in Rheumatic Diseases (BIOBADASER) [19], the Danish Database for Biological Therapies (DANBIO) [20], the National Register for Biological Treatment in Finland (ROB-FIN) [21], and the Norway Disease Modifying AntiRheumatic Drugs Register (NOR-DMARD) [22].

In determining the long-term safety and effectiveness of bDMARDs for AS, it is important to consider the potential confounding effects of other comorbid conditions. Unless comorbid conditions are considered, adverse events may be incorrectly attributed to bDMARD therapy. Comorbidity has been shown to be a significant predictor of health outcomes in patients with rheumatoid arthritis [23, 24], with premature mortality largely attributed to cardiovascular disease, infection, and malignancy [25]. Several registries, including our own, have reported a high prevalence of comorbid conditions in patients with rheumatoid arthritis commencing bDMARDs $[20,26,27]$ but the prevalence of comorbid conditions in patients with AS commencing bDMARD therapy has not, thus far, been widely reported.

Before bDMARDs can be prescribed under governmentsubsidized schemes in Australia certain stringent criteria must be met. These include fulfilment of the modified New York criteria for definite AS [28] and failure to respond, over a three-month period, to two or more NSAIDs and an exercise program. Failure to respond is defined as a Bath Ankylosing Spondylitis Disease Activity Index (BASDAI) of at least 4.0 and raised inflammatory markers (erythrocyte sedimentation rate (ESR) $>25 \mathrm{~mm} /$ hour and/or C-reactive protein $(\mathrm{CRP})>10.0 \mathrm{mg} / \mathrm{L}$ ) [29]. Similarly restrictive criteria exist in the UK [30].

In contrast to some other countries [31], previous and/or current malignancy is not an absolute contraindication to prescribing bDMARDs in Australia although increased vigilance is recommended for patients who have a history of malignancy [32]. The incidence and types of malignancies that occur in Australia differ somewhat from those reported elsewhere providing an added imperative to collect Australian data rather than rely on the observations from other registries. For example, Australia has the highest incidence of both melanoma and nonmelanoma skin cancers (NMSCs) in the world attributed to the outdoor lifestyle of a predominantly fair-skinned population and more recently to the reduced ozone layer in this region [33, 34]. It also has a comparatively higher risk of skin cancer in organ transplant recipients who also receive immunosuppressive agents [35] and we have previously reported a threefold increase in the risk of melanoma in Australian methotrexatetreated patients with rheumatoid arthritis compared with the general population [36].

The aim of this study was to describe the baseline characteristics including comorbidities of a populationbased cohort of Australian patients with AS participating in ARAD who are commencing bDMARDs and to compare them to the baseline characteristics of participants enrolled in published bDMARD RCTs in AS.

\section{Methods}

2.1. ARAD Design. The structure and governance of ARAD has been described previously [17]. Briefly, ARAD was established by the Australian Rheumatology Association (ARA) in 2003 as a voluntary registry to collect longitudinal health outcomes data from Australian patients with inflammatory arthritis treated with bDMARDs. Recruitment of controls (those not prescribed biologics) commenced in 2000 and is ongoing although numbers are small to date. The primary goal of ARAD is to determine the long-term effectiveness and safety of bDMARDs in routine clinical practice.

Ethical approval has been granted by the Institutional Human Research Ethics Committees of Cabrini Hospital, Melbourne; Northern Sydney Health; South Eastern Sydney and Illawarra Area Health; Australian Government Department of Veterans Affairs; Monash University; Royal Children's Hospital, Melbourne; NSW Population and Health Services Research Ethics; Tasmanian Human Research Ethics Committee; Australian Institute of Health and Welfare; Cancer Institute of NSW; Department of Health (CHIC) Western Australia; St Vincent's Hospital, Melbourne; The Children's Hospital, Westmead; Department of Health South Australia Human Research Ethics Committee; Queensland Cancer Registry; the Tasmanian Cancer Registry; the Victorian Cancer Registry; the ACT Cancer Registry; Northern Territory Cancer Registry, the New South Wales Central Cancer Registry, the Western Australian Cancer Registry, and the National Cancer Statistics Clearing House. All participants provide written informed consent.

2.2. Data Collection. Data collected from the rheumatologist at baseline includes the TNF alpha inhibitor prescribed, ESR, CRP, and the Bath Ankylosing Spondylitis Disease Activity Index (BASDAI) [37]. The BASDAI is a quick, reliable, selfadministered instrument used to determine disease activity in patients with AS. It measures severity of fatigue, spinal and peripheral joint pain, localized tenderness, and morning stiffness and is scored from 0 to $10(0=$ best $)$.

All ARAD participants complete a detailed entry questionnaire and six-month followup questionnaires returned in a reply paid envelope. Returned data are scanned into the database and subject to rigorous quality control and data validation processes to ensure database quality. Data collected from the participants include: demographic details, disease duration and severity, self-reported past and current 
medical history including cancers and other chronic conditions, use of antirheumatic drugs, smoking, and alcohol history, generic measures of quality of life including the Short Form-36 (SF-36) (subscale scores range 0-100, $100=$ perfect health) [38], and Assessment of Quality of Life (AQoL) (score range $0-1,1=$ full health) [39], and arthritis-specific disability assessed by the Health Assessment Questionnaire modified for spondyloarthropathies (S-HAQ) (score range $0-3,0=$ no disability) [40].

For the purpose of this study, the baseline characteristics and health-related quality of life of AS patients commencing bDMARDS were extracted from the last questionnaire completed prior to commencing bDMARD therapy. For participants who enrolled in ARAD after starting therapy, BASDAI, ESR, and CRP at the time of starting therapy were obtained from the referring rheumatologist and comorbidities were only considered if data were available either prior to or within 6 months of the start of bDMARDS. Health-related quality of life data prior to commencing bDMARD therapy was unavailable for these participants.

2.3. Verification of Malignancy. We verified all self-reported malignancies identified prior to commencement of bDMARDs by record linkage to the National Cancer Statistics Clearing House (NCSCH) and the Victorian State Cancer Registry (VCR) in 2007. The NCSCH is a repository of data for each state cancer registry in Australia apart from the state of Victoria. Since 1982 the state registries have collected details of all malignancies apart from nonmelanocytic skin cancers, and notification of malignancy to the relevant state registry is mandatory by law. Virtual complete ascertainment is achieved by notification from pathology laboratories, hospital medical record departments, and by screening of death certificates. The International Classification of Diseases-9th Revision (ICD-9) is used to code site of malignancy [41] and the ICD-O morphology rubrics to code histological type $[42,43]$. At the time of the study the NCSCH and VCR were complete up until the end of 2003 and 2005, respectively. Self-reported malignancies prior to 1982 and after 2003/2005 for the NCSCH and VCR, respectively, and all self-reported nonmelanocytic skin cancers were verified by obtaining pathology reports and/or confirmation by the treating doctor.

2.4. Identification of Published AS RCTs of bDMARDs. A PubMed search was conducted using the terms "ankylosing spondylitis" and the names of the three TNF inhibitors individually "infliximab" or "etanercept" or "adalimumab" to identify relevant AS RCTs of bDMARDs. Relevant baseline participant data were extracted from the RCTs and weighted means calculated for comparison with the ARAD data.

2.5. Data Analysis. Responses to the health related quality of life instruments (SF-36, EuroQoL and AQoL) were coded according to the standard published algorithms as described by the developers $[38,39,44]$. Independent sample $t$-tests were used to compare data in ARAD with that reported in
TABLE 1: Baseline demographic and clinical characteristics of AS patients enrolled in ARAD who have been exposed to biological therapy $(n=354)^{*}$.

\begin{tabular}{lc}
\hline Mean (SD) age, years & $45.1(12.3)$ \\
Males (\%) & $254(71.8)$ \\
Mean (SD) disease duration, years $(n=299)$ & $18.5(12.1)$ \\
Mean (SD) delay in diagnosis, years $(n=310)$ & $5.5(7.1)$ \\
Concomitant DMARDs, $N(\%)$ & $72(20.3)$ \\
Methotrexate (oral or IM) & $50(14.1)$ \\
Salazopyrin & $22(6.2)$ \\
Leflunomide & $5(1.4)$ \\
Currently taking prednisolone, $N(\%)$ & $42(11.9)$ \\
Smoking history, $N(\%)$ & \\
Current & $61(17.2)$ \\
Past & $98(27.7)$ \\
Never & $195(55.1)$ \\
Alcohol consumption, $N(\%)$ & \\
Daily & $62(17.5)$ \\
Sometimes & $217(61.2)$ \\
Never & $75(21.2)$ \\
Mean $(S D)$ ESR, mm/hr $(n=274)$ & $35.4(24.8)$ \\
Mean $(S D)$ CRP, mg/L $(n=300)$ & $31.8(31.9)$ \\
BASDAI $(0-10,0=$ best $)(n=301)$ & $7.6(4.5)$ \\
Mean $(S D)$ S-HAQ score $(0-3,0$ no $=$ disability) $* *$ & $0.86(0.60)$ \\
Mean $(S D)$ AQoL score $(0-1,1=\text { full health })^{* *}$ & $0.55(0.25)$ \\
SF-36 score** & \\
Physical component & $36.2(10.6)$ \\
Mental component & $45.1(11.1)$ \\
$*$ unless otherwise indicated & \\
$* * n=198$ in those prior to or within 6 months of commencing bDMARDS \\
\end{tabular}

previously conducted RCTs. All data were analysed using STATA (Version 10.0, Stata Corporation, College Station, Tex, USA).

\section{Results}

To 11th December 2008, 3025 participants were enrolled in ARAD. They included 2366 with rheumatoid arthritis, 389 with AS, 186 with psoriatic arthritis, and 83 with juvenile idiopathic arthritis. 201 (75.2\%) rheumatologists from all Australian states and territories had contributed patients. Thirty-five out of the 389 AS participants (9\%) had never taken bDMARD therapy and were excluded from further analysis. At the time of the analysis, 136 were currently taking etanercept, 124 were taking infliximab, 60 were taking adalimumab $(n=320)$, and 34 were not currently taking bDMARDs.

The baseline characteristics of the 354 AS participants who commenced biological therapy are summarised in Table 1.

At the time of commencement of bDMARD therapy, $72(20.3 \%)$ participants were taking at least one DMARD (65 were taking one DMARD and 7 were taking two), most commonly methotrexate $(n=50,14.1 \%)$ or salazopyrin 


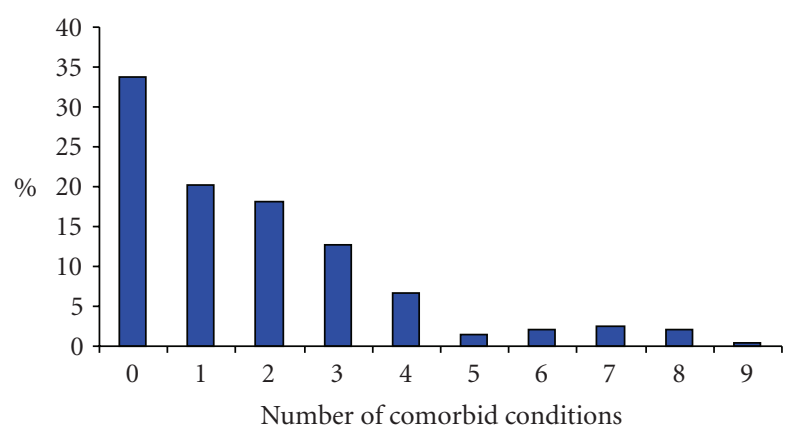

FIgURE 1: Number of comorbid conditions in patients with ankylosing spondylitis commencing biological therapy $(n=198)$.

TABle 2: Self-reported comorbid conditions among ankylosing spondylitis patients commencing biological therapy $(n=198)$.

\begin{tabular}{lc}
\hline Condition & $N(\%)$ \\
\hline Gastrointestinal disease & $62(31.3)$ \\
Hypertension & $51(25.8)$ \\
Eye disorder & $32(16.1)$ \\
Hypercholesterolaemia & $31(15.6)$ \\
Depression & $28(14.1)$ \\
Anaemia or other blood disorder & $28(14.1)$ \\
Lung disease & $21(10.6)$ \\
Neurological disorder & $18(9.0)$ \\
Osteoporosis & $17(8.6)$ \\
Alcohol and drug & $13(6.6)$ \\
Other heart disease & $13(6.5)$ \\
Liver disease & $7(3.5)$ \\
Thyroid disorder & $7(3.5)$ \\
Diabetes Mellitus & $7(3.5)$ \\
Cerebrovascular accident & $5(2.5)$ \\
Kidney disease & $4(2.0)$ \\
Angina & $4(2.0)$ \\
Mental illness other than depression & $4(2.0)$ \\
Tuberculosis & $2(1.0)$ \\
\hline
\end{tabular}

( $n=22,6.2 \%)$; while $42(11.9 \%)$ participants were taking prednisolone. Participants had evidence of active disease (mean (SD) BASDAI score 7.6 (4.5). Of those with AS who had ever taken bDMARDs $(n=354)$, quality of life data at baseline was available for 198 (56\%) who had enrolled in ARAD prior to or within 6 months of commencing bDMARDs. They had moderate disability (mean (SD) SHAQ 0.86 (0.60) and impaired quality of life, mean (SD) AQoL score 0.55 (0.25); SF-36 Physical Component score 36.2 (10.6), SF-36 Mental Component score 45.1 (11.1)).

At least one comorbid condition (past or current) was reported by $131(66.1 \%)$ participants and $91(46.0 \%)$ reported more than one (Figure 1).

The most frequently self-reported comorbidities were gastrointestinal disease reported by 61 (31.3\%) participants, hypertension $51(25.8 \%)$, eye disorders 32 (16.1\%), dyslipidaemia 31 (15.6\%), and depression 28 (14.1\%) (Table 2).
Twenty four participants $(6.8 \%)$ had a previous malignancy: nonmelanoma skin cancer $(n=15)$, melanoma $(n=$ $4)$, prostate cancer $(n=2)$, breast cancer $(n=1)$, cervical cancer $(n=1)$, and bowel cancer $(n=1)$.

We identified 4 published RCTs of bDMARDs in AS (Table 3) [11, 14-16]. Compared with participants in previous RCTs, there was a similar proportion of males $(71.8 \%$ versus $73.8 \%, P=.46)$, but ARAD participants were older (mean (SD) age 45.1 (12.3) years versus 41.9 (6.0) years, $P<$ .001 ), had a longer disease duration (mean (SD) duration 18.5 (12.1) years versus $12.6(5.0)$ years, $P<.001)$ and had higher baseline BASDAI scores (mean (SD) 7.6 (4.5) versus 4.1 (0.83), $P<.001)$.

\section{Discussion}

We have described the baseline comorbidities of a population-based cohort of patients with AS commencing biologic therapy extracted from a national biologic registry. As $75 \%$ of Australian rheumatologists contribute patients to ARAD and as all states and territories are represented, we think that our patient sample is representative of the Australian population. Two thirds of our sample reported having at least one comorbid condition, almost half (46.0\%) had more than one, and $6.8 \%$ had a history of malignancy. In comparison with participants in RCTs of bDMARDs for AS, ARAD AS participants commencing biological therapy were older, had longer duration of disease, and had more severe disease at the time of commencement of bDMARDs. They also had significant disability and impaired quality of life.

To put the high level of baseline comorbidity into context, Figure 2 shows comparable data for ARAD participants with rheumatoid arthritis (RA) commencing biologics. Despite the younger age of the AS cohort (mean age 45.1 (12.3) versus 57.0 (12.5) years for the RA cohort, $P=$ .001) the prevalence of at least one comorbid condition was comparable [27] and it is also comparable to the reported baseline comorbidity of RA patients commencing bDMARDs elsewhere [23, 26].

The significantly greater disease activity at commencement of bDMARDs in our AS cohort compared with RCTs [11, 14-16] most likely reflects the stringent PBS requirements for approval of bDMARDs for AS in Australia. Our patients also appear to have greater disease activity at bDMARD commencement than patients commencing bDMARDs in routine care in other settings. For example, a Spanish study of patients with AS commencing biologic therapy reported mean BASDAI scores of 4.5 (versus 7.1 in our study) [45].

Comparable efficacy between RCTs and clinical practice is hardly ever achieved due in part to patient selection, differences in comedications and comorbidities and treatment adherence [46]. Participants in RCTs are likely to be different in some important respects to individual patients seen in clinical practice since minority groups, older individuals, and those at risk of adverse events may be deliberately excluded. The differences we observed may also have been 


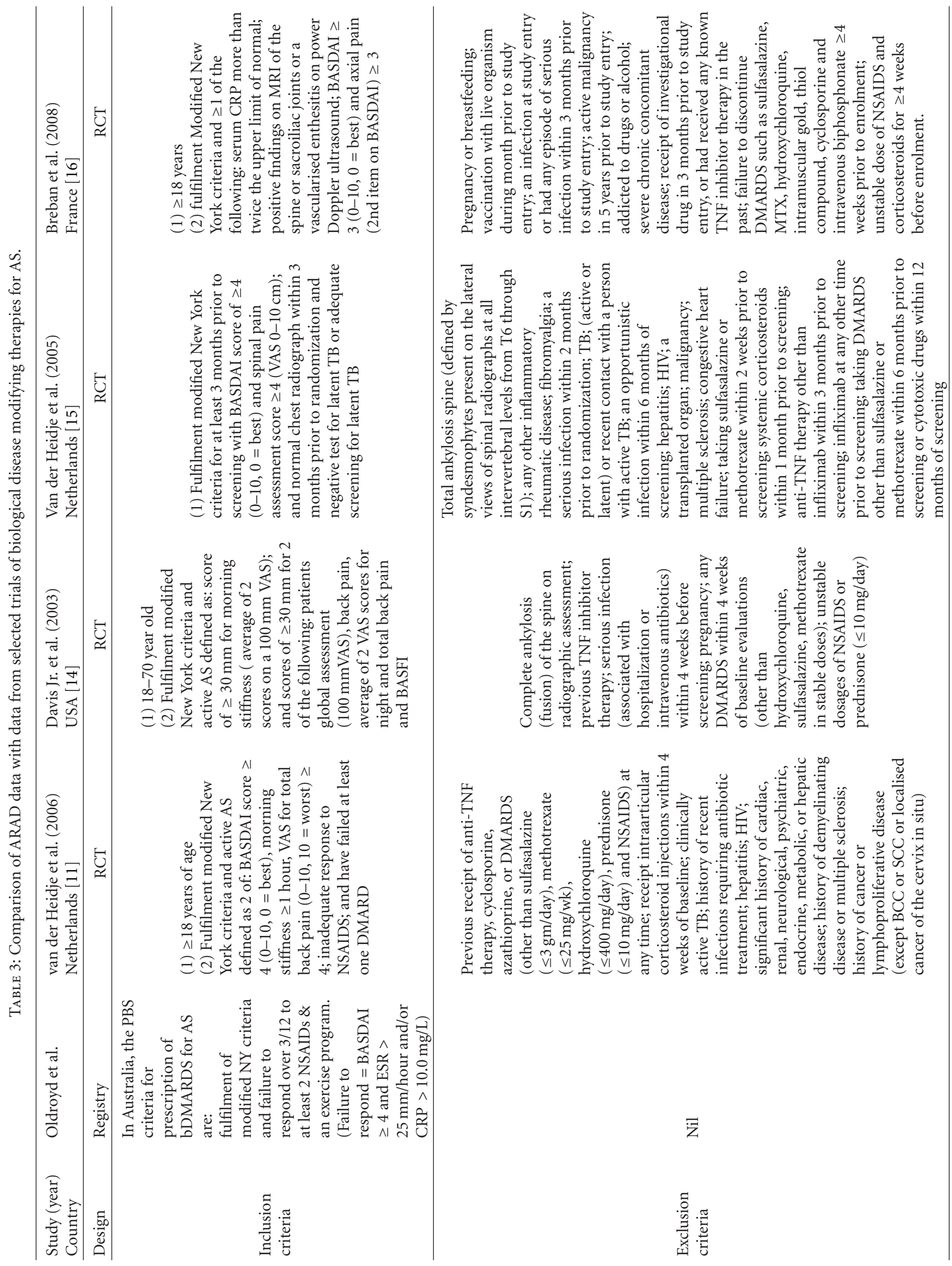




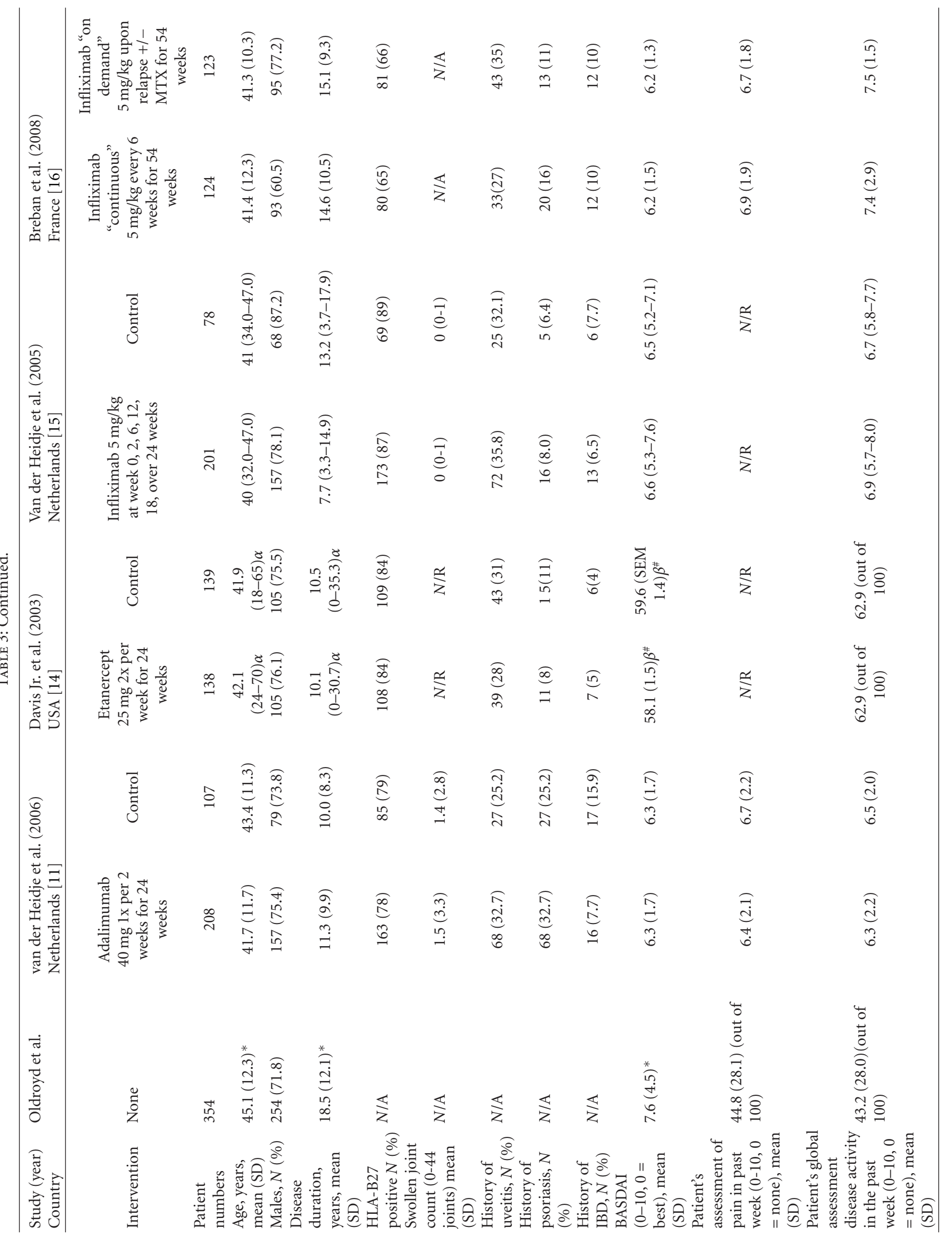




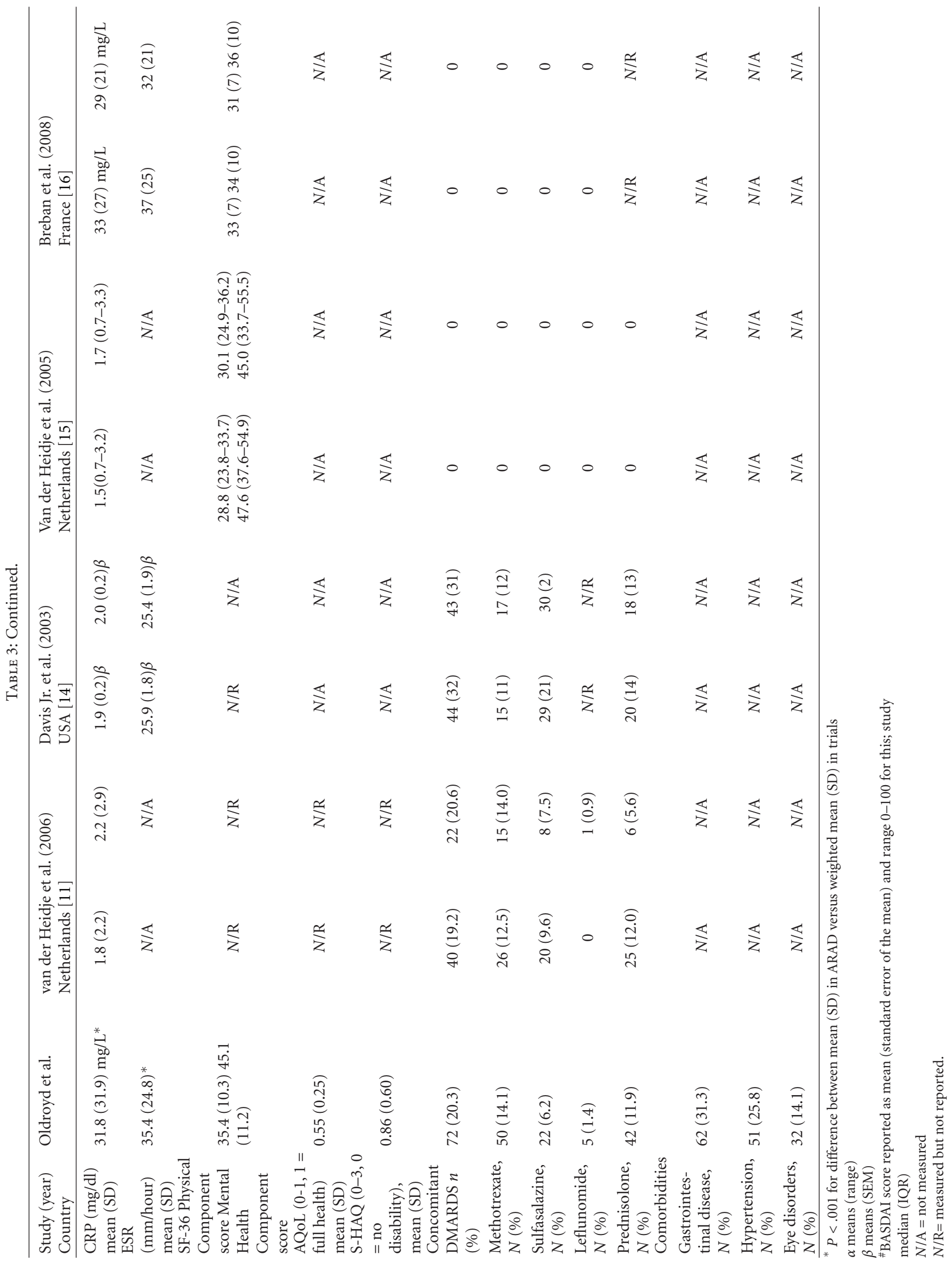




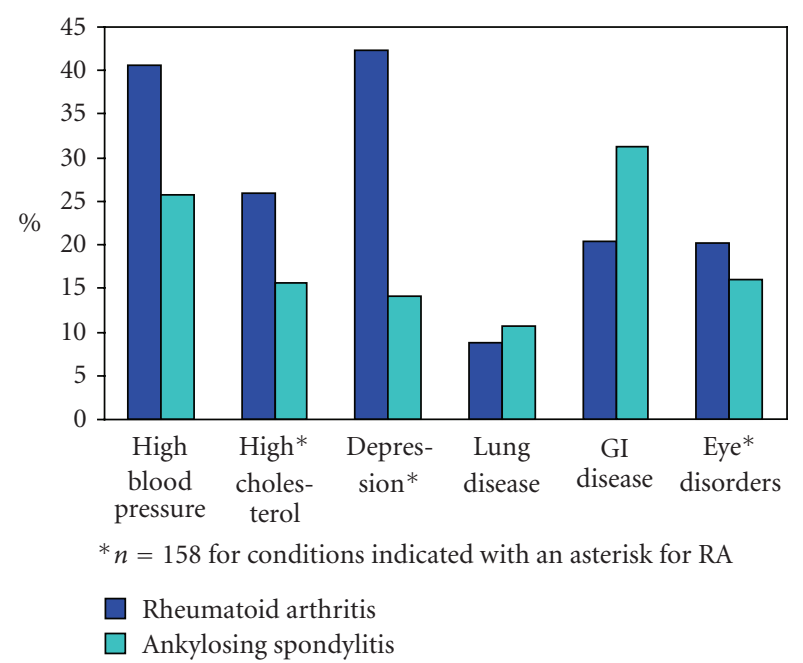

FIGURE 2: Self-reported comorbidities in ankylosing spondylitis $(n=198)$ and rheumatoid arthritis $\left(N=624^{*}\right)$.

accentuated by the lack of available treatment options prior to the introduction of bDMARD therapy for AS. Over time, it is likely that the average age and disease duration of those prescribed bDMARDs will diminish as newly diagnosed patients who fulfil criteria are treated earlier.

Two of the four trials excluded participants with a history of malignancy $[11,15]$ and a third excluded patients with an active malignancy in the 5 years prior to the study [16]. The fourth trial did not specify malignancy as an exclusion criterion, and no details regarding presence/absence of malignancy in participants at baseline were reported [14]. We found that $24(6.8 \%)$ AS patients commencing bDMARD therapy in our study had a verified history of malignancy including 15 with nonmelanoma skin cancer and 4 with melanoma. The true prevalence of nonmelanoma skin cancers is likely to be higher but this data is not captured by Australian state registries and we only included self-reported cancers that we could verified by pathology or doctor report.

Available data on risk of malignancy in AS are limited and mainly confined to the increased cancer risk observed in patients with AS subjected to radiation treatment [47]. Recent Swedish population-based studies have not found an increased risk of lymphoma or malignancies overall [48, 49]. It is also unknown whether biologic therapy confers any increased risk of malignancy or recurrence of malignancy in AS. These risks may only be determined by careful long-term comparisons of exposed and unexposed populations.

There are several potential limitations of our study. While we were able to verify the validity of self-reported malignancies, we did not verify the validity of self-report of comorbid conditions. As reported previously, ARAD data are derived predominantly from patient questionnaire [17]. At the time that ARAD was established, there were concerns about the administrative burden of applying for biological therapy and rheumatologists were asked to provide a minimum of information at baseline only. Nevertheless, baseline comorbidities reported by patients with RA in
ARAD are comparable to those reported in other studies [18].

A second limitation of our study is that we have not routinely collected data about the presence/absence of extra spinal features of AS such as uveitis, psoriasis, and inflammatory bowel disease, due in part to the fact that ARAD was originally set up for RA. It is likely that some self-reported comorbidities in our study are likely to relate to these extraspinal manifestations of the disease.

\section{Conclusion}

We found that a population-based cohort of patients with AS commencing biological therapy and participating in a national biologics registry already has significant comorbidities. Despite their younger age, the prevalence of comorbidities was comparable to that seen in a populationbased RA cohort commencing bDMARDs. These findings have important implications for monitoring patients while on therapy, assessing the long-term health outcomes of bDMARD therapy and attribution of adverse events. We also found that participants in RCTs of bDMARDs for AS were not representative of Australian patients with AS commencing bDMARDs in routine care. As well as being older, having longer disease duration, and more active disease, $6.8 \%$ also had a history of verified malignancy. These findings highlight the importance of systematically collecting postmarketing longitudinal outcome data for bDMARDs in routine care.

\section{Acknowledgments}

The authors gratefully acknowledge their funding sources: National Health and Medical Research Council (NMHRC) Enabling Grant 384330 (2006-2010), Monash University (2006-2010) and unrestricted educational grants from Abbott Australasia Pty Ltd (2003-2008), Amgen Australia Pty Ltd (2003-2007), Roche Products Pty Ltd (2008), Wyeth Australia Pty Ltd (2003-2008), Sanofi-Aventis Australia Pty Ltd (2003-2004), and Schering-Plough Pty Ltd (20032004) paid to the Australian Rheumatology Association. They would also like to acknowledge the contributions of Chris Reid, Lyndall Henderson, Rosemarie van den Haak, Nino Hay, the participating rheumatologists, and the ARAD Scientific Advisory Committee (Jonathan Akikusa, Claire Barrett, Andrea Bendrups, Ann Dorai Raj, Graeme Carroll, Catherine Hill, Kevin Murray, Judith Nguyen, Kevin Pile, Ian Portek, and Jane Zochling).

\section{References}

[1] British Society for Rheumatology, "BSR guideline for prescribing TNF-alpha blockers in adults with ankylosing spondylitis," December 2005, http://www.rheumatology.org.uk.

[2] J. C. Davis, D. van der Heijde, M. Dougados, and J. M. Woolley, "Reductions in health-related quality of life in patients with ankylosing spondylitis and improvements with etanercept therapy," Arthritis Care and Research, vol. 53, no. 4, pp. 494501, 2005. 
[3] M. M. Ward, "Health-related quality of life in ankylosing spondylitis: a survey of 175 patients," Arthritis Care and Research, vol. 12, no. 4, pp. 247-255, 1999.

[4] K. Lehtinen, "Mortality and causes of death in 398 patients admitted to hospital with ankylosing spondylitis," Annals of the Rheumatic Diseases, vol. 52, no. 3, pp. 174-176, 1993.

[5] H. Dagfinrud, K. B. Hagen, and T. K. Kvien, "Physiotherapy interventions for ankylosing spondylitis," Cochrane Database of Systematic Reviews, no. 1, Article ID CD002822, 2008.

[6] M. Dougados, R. Caporal, P. Doury, et al., "A double blind crossover placebo controlled trial of ximoprofen in as.", Journal of Rheumatology, vol. 16, no. 8, pp. 1167-1169, 1989.

[7] M. Dougados, A. Gueguen, J.-P. Nakache, et al., "Ankylosing spondylitis: what is the optimum duration of a clinical study? A one year versus a 6 weeks non-steroidal anti-inflammatory drug trial," Rheumatology, vol. 38, no. 3, pp. 235-244, 1999.

[8] R. G. W. Lambert, D. Salonen, P. Rahman, et al., "Adalimumab significantly reduces both spinal and sacroiliac joint inflammation in patients with ankylosing spondylitis: a multicenter, randomized, double-blind, placebo-controlled study," Arthritis and Rheumatism, vol. 56, no. 12, pp. 40054014, 2007.

[9] J. Braun, J. Brandt, J. Listing, et al., "Treatment of active ankylosing spondylitis with infliximab: a randomised controlled multicentre trial," The Lancet, vol. 359, no. 9313, pp. 11871193, 2002.

[10] J. D. Gorman, K. E. Sack, and J. C. Davis Jr., "Treatment of ankylosing spondylitis by inhibition of tumor necrosis factor $\alpha$," The New England Journal of Medicine, vol. 346, no. 18, pp. 1349-1356, 2002.

[11] D. van der Heijde, A. Kivitz, M. H. Schiff, et al., "Efficacy and safety of adalimumab in patients with ankylosing spondylitis: results of a multicenter, randomized, double-blind, placebocontrolled trial," Arthritis and Rheumatism, vol. 54, no. 7, pp. 2136-2146, 2006.

[12] J. Zochling, D. van der Heijde, M. Dougados, and J. Braun, "Current evidence for the management of ankylosing spondylitis: a systematic literature review for the ASAS/EULAR management recommendations in ankylosing spondylitis," Annals of the Rheumatic Diseases, vol. 65, no. 4, pp. 423-432, 2006.

[13] J. Brandt, A. Khariouzov, J. Listing, et al., "Six-month results of a double-blind, placebo-controlled trial of etanercept treatment in patients with active ankylosing spondylitis," Arthritis and Rheumatism, vol. 48, no. 6, pp. 1667-1675, 2003.

[14] J. C. Davis Jr., D. van der Heijde, J. Braun, et al., "Recombinant human tumor necrosis factor receptor (etanercept) for treating ankylosing spondylitis: a randomised, controlled trial," Arthritis and Rheumatism, vol. 48, no. 11, pp. 32303236, 2003.

[15] D. van der Heijde, B. Dijkmans, P. Geusens, et al., "Efficacy and safety of infliximab in patients with ankylosing spondylitis: results of a randomized, placebo-controlled trial (ASSERT)," Arthritis and Rheumatism, vol. 52, no. 2, pp. 582-591, 2005.

[16] M. Breban, P. Ravaud, P. Claudepierre, et al., "Maintenance of infliximab treatment in ankylosing spondylitis: results of a one-year randomized controlled trial comparing systematic versus on-demand treatment," Arthritis and Rheumatism, vol. 58, no. 1, pp. 88-97, 2008.

[17] R. Buchbinder, L. March, M. Lassere, et al., "Effect of treatment with biological agents for arthritis in Australia: the Australian Rheumatology Association Database," Internal Medicine Journal, vol. 37, no. 9, pp. 591-600, 2007.
[18] A. Silman, D. Symmons, D. G. I. Scott, and I. Griffiths, "British society for rheumatology biologics register," Annals of the Rheumatic Diseases, vol. 62, supplement 2, pp. 28-29, 2003.

[19] L. Carmona and J. J. Gomez-Reino, "On behalf of the BIOBADASER Group. Survival of TNF antagonists in spondylarthritis is better than in rheumatoid arthritis. Data from the Spanish registry BIOBADASER," Arthritis Research \& Therapy, vol. 8, p. R72, 2006.

[20] M. L. Hetland, "DANBIO: a nationwide registry of biological therapies in Denmark," Clinical and Experimental Rheumatology, vol. 23, supplement 5, pp. S205-S207, 2005.

[21] L. Konttinen, R. Tuompo, T. Uusitalo, et al., "Anti-TNF therapy in the treatment of ankylosing spondylitis: the Finnish experience.," Clinical Rheumatology, vol. 26, no. 10, pp. 16931700, 2007.

[22] T. K. Kvien, M. S. Heiberg, E. Lie, et al., "A Norwegian DMARD register: prescriptions of DMARDs and biological agents to patients with inflammatory rheumatic diseases," Clinical and Experimental Rheumatology, vol. 23, supplement 5, pp. S188-S194, 2005.

[23] I. Rupp, H. C. Boshuizen, C. E. Jacobi, H. J. Dinant, and G. A. M. van den Bos, "Comorbidity in patients with rheumatoid arthritis: effect on health-related quality of life," Journal of Rheumatology, vol. 31, no. 1, pp. 58-65, 2004.

[24] L. Carmona, M. A. Descalzo, E. Perez-Pampin, et al., "Allcause and cause-specific mortality in rheumatoid arthritis are not greater than expected when treated with tumour necrosis factor antagonists," Annals of the Rheumatic Diseases, vol. 66, no. 7, pp. 880-885, 2007.

[25] F. Wolfe, D. M. Mitchell, J. T. Sibley, et al., "The mortality of rheumatoid arthritis," Arthritis and Rheumatism, vol. 37, no. 4, pp. 481-494, 1994.

[26] K. Hyrich, D. Symmons, K. Watson, and A. Silman, "Baseline comorbidity levels in biologic and standard DMARD treated patients with rheumatoid arthritis: results from a national patient register," Annals of the Rheumatic Diseases, vol. 65, no. 7, pp. 895-898, 2006.

[27] A. M. Briggs, L. March, M. Lassere, et al., "Baseline comorbidities in an Australian population-based cohort of rheumatoid arthritis patients receiving biological therapy: data from the Australian Rheumatology Association Database (ARAD)," International Journal of Rheumatology, in press.

[28] S. van der Linden, H. A. Valkenburg, and A. Cats, "Evaluation of diagnostic criteria for ankylosing spondylitis. A proposal for modification of the New York criteria," Arthritis and Rheumatism, vol. 27, no. 4, pp. 361-368, 1984.

[29] PBS. Pharmaceutical Benefits Scheme, Australian Government Department of Health and Aging, January 2007, http://www.pbs.gov.au/html/home.

[30] National Institute for Health and Clinical Excellence, "Adalimumab, etanercept and infliximab for the treatment of ankylosing spondylitis," June 2008, http://www .nice.org.uk/nicemedia/pdf/TA143QuickRefGuide.pdf.

[31] National Institute for Clinical Excellence, Guidance on the Use of Etanercept and Infliximab for the Treatment of Rheumatoid Arthritis, NICE, London, UK, 2002.

[32] L. C. Perera, K. E. Tymms, B. J. Wilson, et al., "Etanercept in severe active rheumatoid arthritis: first Australian experience," Internal Medicine Journal, vol. 36, no. 10, pp. 625-631, 2006.

[33] M. P. Staples, M. Elwood, R. C. Burton, J. L. Williams, R. Marks, and G. G. Giles, "Non-melanoma skin cancer in Australia: the 2002 national survey and trends since 1985," Medical Journal of Australia, vol. 184, no. 1, pp. 6-10, 2006. 
[34] D. M. Parkin, S. L. Whelan, J. Ferlay, et al., Cancer Incidence in Five Continents, vol. 8, International Agency for Research on Cancer, Lyon, France, 2002.

[35] J. N. Bouwes Bavinck, D. R. Hardie, A. Green, et al., “The risk of skin cancer in renal transplant recipients in Queensland, Australia: a follow-up study," Transplantation, vol. 61, no. 5, pp. 715-721, 1996.

[36] R. Buchbinder, M. Barber, L. Heuzenroeder, et al., "Incidence of melanoma and other malignancies among rheumatoid arthritis patients treated with methotrexate," Arthritis Care and Research, vol. 59, no. 6, pp. 794-799, 2008.

[37] S. Garrett, T. Jenkinson, L. G. Kennedy, H. Whitelock, P. Gaisford, and A. Calin, "A new approach to defining disease status in ankylosing spondylitis: the bath ankylosing spondylitis disease activity index," Journal of Rheumatology, vol. 21, no. 12, pp. 2286-2291, 1994.

[38] J. Ware, M. Kosinski, and S. Keller, SF-36 Physical and Mental Health Summary Scales: A User's Manual, New England Medical Centre, The Health Institute, Boston, Mass, USA, 1994.

[39] G. Hawthorne, J. Richardson, and R. Osborne, "The Assessment of Quality of Life (AQoL) Instrument: a psychometric measure of health-related quality of life," Quality of Life Research, vol. 8, no. 3, pp. 209-224, 1999.

[40] L. H. Daltroy, M. G. Larson, W. N. Roberts, and M. H. Liang, "A modification of the Health Assessment Questionnaire for the spondyloarthropathies," Journal of Rheumatology, vol. 17, no. 7, pp. 946-950, 1990.

[41] World Health Organization, International Classification of Disease, WHO, Geneva, Switzerland, 1977.

[42] World Health Organization, International Classification of Diseases for Oncology, WHO, Geneva, Switzerland, 1976.

[43] R. Cote, Ed., Systematized Nomenclature of Medicine, College of American Pathologists, Skokie, Ill, USA, 1980.

[44] M. Oppe, R. Rabin, and F. de Charro, "On behalf of the EuroQoL Group 2007. EQ-5D User Guide,” February 2008, http://www.euroqol.org.

[45] R. Ariza-Ariza, B. Hernandez-Cruz, and F. Navarro-Sarabia, "Physical function and health-related quality of life of Spanish patients with ankylosing spondylitis," Arthritis Care and Research, vol. 49, no. 4, pp. 483-487, 2003.

[46] W. Kievit, J. Fransen, A. J. M. Oerlemans, et al., "The efficacy of anti-TNF in rheumatoid arthritis, a comparison between randomised controlled trials and clinical practice," Annals of the Rheumatic Diseases, vol. 66, no. 11, pp. 1473-1478, 2007.

[47] H. A. Weiss, S. C. Darby, and R. Doll, "Cancer mortality following X-ray treatment for ankylosing spondylitis," International Journal of Cancer, vol. 59, no. 3, pp. 327-338, 1994.

[48] N. Feltelius, A. Ekbom, and P. Blomqvist, "Cancer incidence among patients with ankylosing spondylitis in Sweden 1965-1995: a population based cohort study," Annals of the Rheumatic Diseases, vol. 62, no. 12, pp. 1185-1188, 2003.

[49] J. Askling, L. Klareskog, P. Blomqvist, M. Fored, and N. Feltelius, "Risk for malignant lymphoma in ankylosing spondylitis: a nationwide Swedish case-control study," Annals of the Rheumatic Diseases, vol. 65, no. 9, pp. 1184-1187, 2006. 


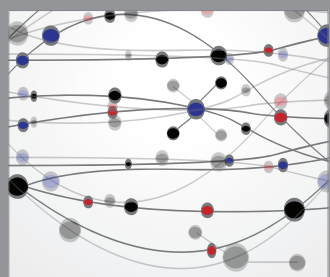

The Scientific World Journal
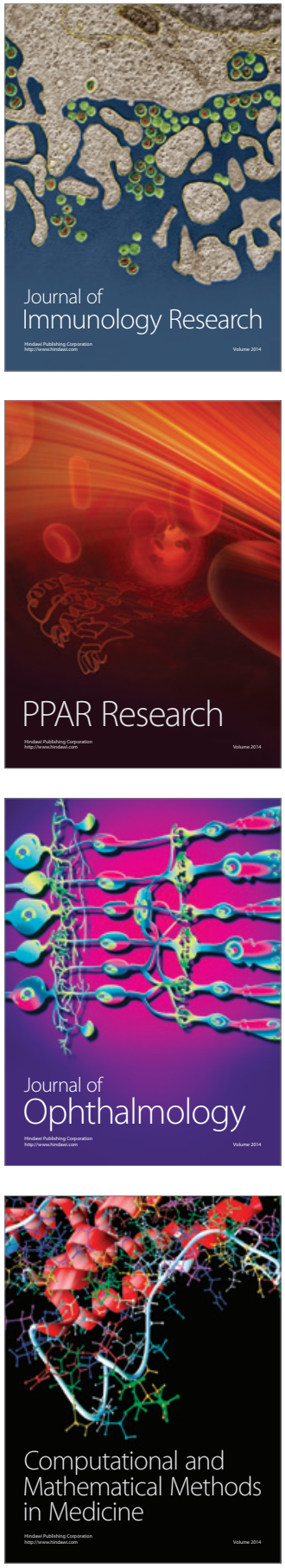

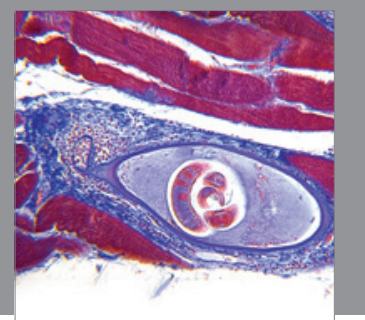

Gastroenterology

Research and Practice
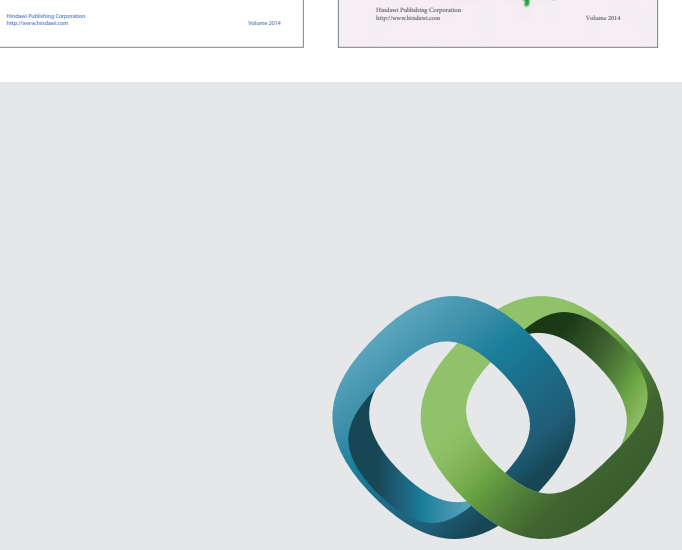

\section{Hindawi}

Submit your manuscripts at

http://www.hindawi.com
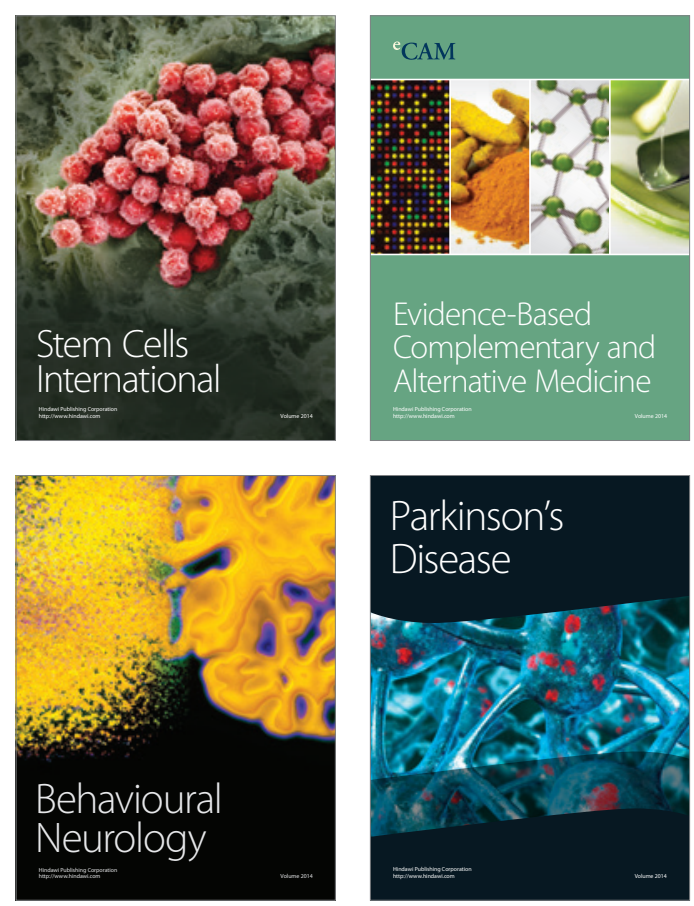

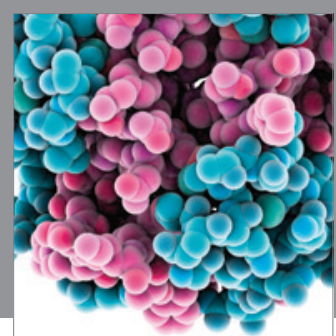

Journal of
Diabetes Research

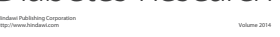

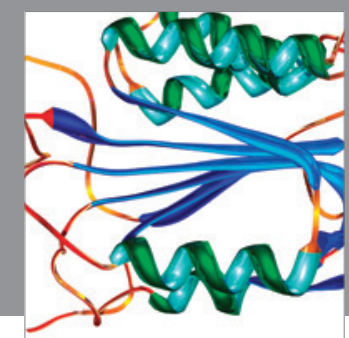

Disease Markers
